\title{
SINOPSE DA FLORA MEDICINAL DO CARIRI PARAIBANO
}

\author{
Maria de Fátima Agra ${ }^{1 *}$, George Sidney Baracho ${ }^{2}$, Ionaldo J. Diniz Basílio ${ }^{1}$, Kiriaki Nurit ${ }^{1}$, Victor \\ Peçanha Coelho ${ }^{1}$ \& Danila de Araújo Barbosa ${ }^{1}$ \\ ${ }^{1}$ Laboratório de Tecnologia Farmacêutica, Universidade Federal da Paraíba, Caixa Postal 5009, 58015-970 João Pessoa, Paraíba, Brasil. \\ ${ }^{2}$ Laboratório de Biologia Vegetal, Departamento de Botânica, Instituto de Ciências Biológicas, Universidade de Pernambuco, Rua Arnóbio Marques \\ 310, Santo Amaro, 50100-130 Recife, Pernambuco Brasil. \\ *E.mail: agramf@ltf.ufpb.br
}

\section{RESUMO}

Realizou-se um levantamento da diversidade das plantas de usos medicinais na flora do Cariri Paraibano, Brasil. Foram registrados os usos etnomedicinais de 70 espécies, pertencentes a 59 gêneros e 32 famílias. Destas, 17 famílias estão representadas por apenas uma espécie, correspondendo a 53\% do total. As famílias com maior número de espécies são Fabaceae s. l., (12 espécies), Solanaceae (seis), Euphorbiaceae (cinco), Asteraceae (quatro) e Bromeliaceae e Convolvulaceae, ambas com três espécies cada. A diversidade florística obtida neste levantamento está representada principalmente por espécies de Angiospermas e apenas Selaginella convoluta Spreng, pertence ao grupo das Pteridófitas. Cerca de 60 espécies, que correspondem a 86\%, foram referidas para mais de uma doença e outras 10 para apenas um uso. Dentre as espécies com mais de um uso, destaca-se Amburana cearensis (Allemão)A.C.Sm., referida para mais de cinco usos medicinais. A maioria das plantas comercializadas e empregadas com fins medicinais é constituída de espécies nativas. Os raizeiros e vendedores possuem considerável conhecimento das plantas medicinais e seus usos para uma ampla diversidade de doenças, principalmente, pela experiência adquirida de gerações anteriores.

Palavras-chave: Caatinga, Cariri da Paraíba, etnomedicina, plantas medicinais, saber local.

\section{ABSTRACT}

SYNOPSIS OF THE MEDICINAL FLORA OF “CARIRI PARAIBANO”. A survey on the diversity of medicinal plants in the flora of the "Cariri Paraibano", Brazil, has been made. 70 plant species belonging to 59 genera and 32 families were recorded by their use in the popular medicine in the study area. Of these, seventeen families are represented by only one species, which correspond to about 53\% of the total. The families with highest number of species are Fabaceae s. l. (12 species), Solanaceae (six species), Euphorbiaceae (five species), Asteraceae (four species), and Bromeliaceae and Convolvulaceae, both with three species each. The floristic diversity found in this survey is dominated by Angiosperms and only one species, Selaginella convoluta Spreng, belongs to the Pteridophytes group. About 60 species, corresponding to $86 \%$, were indicated for more than one disease and ten species for only one. Amburana cearensis (Allemão) A.C.Sm. was indicated for more than five ethnomedicinal uses. The majority of commercialized plants used as medicinal is represented by native species. The "raizeiros" and vendors of medicinal plants have considerable knowledge about them as well as their uses for a wide diversity of diseases, which were obtained mainly from previous generations.

Key words: Caatinga, Cariri da Paraíba, etnomedicine, medicinal plants, local knowledge.

\section{INTRODUÇÃO}

A microrregião do Cariri Paraibano está situada no Platô da Borborema entre $7^{\circ}$ e $8^{\circ} 30^{\prime}$ S e $36^{\circ}$ e $37^{\circ} 30^{\prime} \mathrm{W}$. Localizada na caatinga, caracteriza-se pelo clima semi-árido, quente e seco, com uma vegetação fortemente xerofítica, constituindo-se em uma das áreas mais secas do Brasil (Andrade-Lima 1981). Apresenta-se como uma mata seca e aberta, verde no inverno. Sua composição florística é heterogênea (Agra 1996, Agra et al. 2007), constituída de espécies caducifólias, espinhosas, muitas vezes áfilas (Carvalho \& Carvalho 1985), compreendendo um grande número de Cactaceae, Bromeliaceae, ervas e arbustos 
anuais das famílias Malvaceae (Melochia spp., Sida galheirensis Ulbr. e Herissantia tiubae (K.Schum.) Brizicky, Commelinaceae (Commelina spp.), Euphorbiaceae (Euphorbia spp., Croton spp.) e algumas espécies arbóreas como Erythrina velutina Willd., Amburana cearensis (Allemão) A.C.Sm., Mimosa spp., Caesalpinia pyramidalis Tul., Ziziphus joazeiro Mart., Aspidosperma pyrifolium Mart. e Spondias tuberosa Arruda, entre outras.

Estudos das plantas de uso na medicina popular do Cariri Paraibano são relativamente recentes, com resultados parciais de informações de etnomedicina (Agra et al. 1994, 1996, 2007, Baracho \& Agra 1995, Agra 1996), farmacobotânica (Silva \& Agra 2002; Silva et al. 2002, Basílio et al. 2003, Leal \& Agra 2005, Coelho et al. 2006) e, mais recentemente, de estudos fitoquímicos (Silva et al. 2004).

Este trabalho é uma parte dos estudos que estão sendo desenvolvidos no Cariri Paraibano com o objetivo de registrar as plantas e seus usos na medicina popular, recuperando e registrando do folclore e da tradição o conhecimento adquirido de gerações anteriores.

\section{MATERIAL E MÉTODOS}

As informações etnomedicinais foram obtidas em trabalho de campo, com 15 viagens efetuadas de março de 2003 a junho de 2007, com entrevistas a oito raizeiros e quatro vendedores dos mercados e feiras populares, nos municípios de Serra Branca e Boa Vista. Os 12 entrevistados, todos com idade acima de 45 anos, possuíam grande conhecimento das plantas locais e seus usos. Dois tipos de entrevistas foram realizados: o primeiro, para obter informações das plantas e seus usos; e o segundo, no ambiente de vegetação para a coleta de plantas. As plantas referidas como medicinais foram coletadas em número de quatro a seis amostras e foram posteriormente depositadas no Herbário Professor Lauro Pires Xavier (JPB) e na coleção de referência do Laboratório de Tecnologia Farmacêutica, ambos da Universidade Federal da Paraíba. A identificação das espécies foi realizada por M. F. Agra e G. S. Baracho. As abreviações dos nomes dos autores estão de acordo com Brummitt \& Powell (1992). As espécies estão relacionadas em ordem alfabética, organizadas por família, com base na classificação do APG (2003), seguidas pelo nome botânico, nome(s) popular(es), e uma breve descrição dos usos etnomedicinais, parte(s) usada(s), forma de preparo e modo de usar.

\section{RESULTADOS E DISCUSSÃO}

Foram registrados os usos de 70 espécies, pertencentes a 59 gêneros e 32 famílias que são empregadas na medicina popular do Cariri Paraibano. Dessas, 17 famílias estão representadas por apenas uma espécie, correspondendo à aproximadamente $53 \%$ do total. Em ordem decrescente, as famílias com maior número de espécies são: Fabaceae s. l., com 12 espécies, Solanaceae (seis), Euphorbiaceae (cinco), Asteraceae (quatro), Bromeliaceae e Convolvulaceae, com três espécies cada. A diversidade florística obtida no levantamento está representada por espécies de Angiospermas e apenas Selaginella convoluta (Arn.) Spring, pertence às Pteridófitas.

Treze espécies destacaram-se como as mais populares e reputadas por suas propriedades medicinais: $\mathrm{Si}$ deroxylon obtusifolium (Humb. ex Roem. \& Schult.) T.D. Penn. subsp. obtusifolium, Amburana cearensis (Allemão) A.C. Sm., Anadenathera colubrina var. cebil (Griseb.) Altschul, Bauhinia cheilantha (Bong.) Steud., Cnidoscolus quercifoius Pohl, Commiphora leptophloeos (Mart.) J.B. Gillett, Egletes viscosa (L.) Less., Erythrina velutina Willd., Licania rigida Benth., Maytenus rigida Mart., Myracrodruon urundeuva Allemão e Operculina hamiltonii (G.Don) D.F. Austin.

Para as 70 espécies registradas um total de 208 indicações foi registrado para 64 diferentes usos medicinais. Os principais usos medicinais estão relacionados ao tratamento das doenças do sistema reprodutivo (17,2\%), seguidos do sistema digestivo $(14,1 \%)$, respiratório $(12,5 \%)$ e urinário $(7,8 \%)$, que correspondem a cerca de $50 \%$ do total de usos.

A maioria das plantas, cerca de 60 espécies (85,7\%), foi indicada para mais de uma doença, e dez espécies foram referidas para apenas um uso. Dentre as espécies com mais de um uso, destacouse Amburana cearensis (Allemão) A.C.Sm. que foi referida para mais de cinco usos medicinais.

No tratamento das doenças do sistema reprodutivo $39,3 \%$ dos usos foram referidos para o tratamento dos problemas menstruais, como amenorréia e dismenorréia, seguido das indicações contra inflamações ovaria- 
nas e prostáticas, com 21,4\% e 14,3\%, respectivamente. Nas doenças do sistema respiratório 38,6\% foram referidas para o tratamento das tosses, seguido das bronquites (22,8\%), gripes (14\%), expectorante (7\%), asmas e coqueluches (3,5\% cada). As principais indicações para o sistema digestivo foram registradas como estomáquico (26,5\%), laxativo (17,6\%), purgativo e antidiarréico (14,7\% cada), e nas doenças hepáticas (11,8\%).

A maioria das plantas foi referida para uso interno, com mais de uma forma de preparação e mais de um uso para a mesma planta. As formas para uso interno, em ordem decrescente, foram registradas como decocto para 56 espécies, infuso para 21 e apenas oito espécies foram referidas para maceração.

Algumas preparações são denominadas "garrafadas”, uma mistura de diferentes partes de plantas, como raízes e cascas de caules, que são colocadas em maceração em uma solução hidroalcoólica com vinho ou cachaça. São indicadas para adultos do sexo masculino, porque para crianças e mulheres não são indicadas receitas que levem álcool em sua composição. Outras são preparadas como xaropes, aos quais são adicionados açúcar ou mel de abelha, e popularmente conhecidas como "lambedores", indicadas para as tosses, coqueluches e tônico, geralmente indicados para crianças porque são mais palatáveis e de mais fácil administração.

Para uso externo, as formas de aplicações foram indicadas, principalmente, para as doenças dermatológicas, sendo seis espécies empregadas como decocto e uma como infuso, para banhos ou lavagens das áreas afetadas, como foi referido para Ipomoea asarifolia (Desr.) Roem. \& Schult. (Convolvulaceae) no tratamento de escabioses e dermatites. Em algumas indicações, parte da planta seca (caule ou raiz) é pulverizada e empregada em uso tópico, geralmente indicado para úlceras externas, como por exemplo, o pó da casca do caule de Maytenus rigida Mart. Uma forma mais rara foi a da inalação direta do pó, no qual parte da planta é pulverizada, registrada para as sementes e casca do caule de Amburana cearensis (Allemão) A. C. Sm., indicada para inalação no tratamento de sinusites e doenças respiratórias.

As partes das plantas mais usadas com fins medicinais foram, em ordem decrescente: folhas (com 25 indicações), caule e casca do caule (33), raiz (16), planta inteira (15), flores e inflorescências (quatro), partes aéreas (quatro), frutos (sete), sementes (cinco), látex (três) e tubérculos (seis). A polpa do caule foi registrada para duas espécies de Cactaceae, Melocactus zehntneri (Britton \& Rose) Luetzelb. e Cereus jamacaru DC.

A posologia do uso das plantas variou de uma dose única, uma colher de sopa três vezes ao dia, para um litro diário de infuso ou decocto, a ser usado até o desaparecimento dos sintomas. O diagnóstico nem sempre é exato, geralmente se baseia nas descrições dos sintomas, como relatado pelos informantes. Isto é evidente na indicação de muitas espécies para mais de um emprego medicinal. Exemplos deste tipo de sobreposição é a citação de espécies indicadas como vermífugo e expectorante simultaneamente, entre outras.

A seguir, são apresentadas as espécies catalogadas no presente estudo, as quais compõem a listagem obtida por meio das entrevistas feitas com os informantes, parceiros desta pesquisa:

\section{Amaranthaceae}

Alternanthera brasiliana (L.) Kuntze [acônito, ervanço]

$\mathrm{O}$ infuso das folhas é indicado contra febres. $\mathrm{O}$ infuso das inflorescências é usado no tratamento das cefaléias, tosses, gripes e como expectorante. $\mathrm{O}$ decocto das raízes é utilizado contra diarréias.

\section{Gomphrena demissa Mart. [capitãozinho]}

O decocto das raízes é empregado no tratamento da esterilidade feminina, amenorréias, inflamações e doenças do ovário.

\section{Anacardiaceae}

Myracrodruon urundeuva Allemão [aroeira]

O decocto da casca do caule é utilizado como antiinflamatório ovariano e, em uso tópico, é indicado contra úlceras externas.

Spondias tuberosa Arruda [imbuzeiro, umbuzeiro]

O decocto da casca do caule é empregado como oftálmico. Os frutos, preparados como suco ou "umbuzada", com açúcar e leite, são usados como tônico geral e fonte de vitaminas.

\section{Apocynaceae}

Aspidosperma pyrifolium Mart. [pereiro, pau-pereiro]

Usa-se um decocto da casca do caule contra inflamações do trato urinário e, externamente, contra dermatites. 


\section{Asteraceae}

Egletes viscosa (L.) Less. [macela-do-campo, macela, macela-da-terra]

O infuso ou decocto das inflorescências, tomado após as refeições, é empregado como estomáquico e anti-diarréico.

Pectis elongata Kunth [alecrim-do-mato, alecrimbravo, cuminho-bravo]

Usa-se o infuso da planta inteira como estomáquico e contra hipertensão. $\mathrm{O}$ infuso ou xarope das folhas são empregados contra gripes e tosses.

Pectis oligocephala var. affinis (Gardner) Baker [alecrim-do-mato, alecrim-bravo]

As folhas e a planta inteira apresentam os mesmos usos e indicações da espécie anterior.

Tagetes minuta L. [coari-bravo, cravo-de-viúva, cravobravo]

O infuso ou decocto das folhas é empregado como sedativo, febrífugo e contra amenorréias. O suco em jejum é usado no tratamento das verminoses.

\section{Bignoniaceae}

Tabebuia aurea (Silva-Manso) Benth. \& Hook.f. ex

S. Moore [craibeira]

O decocto ou xarope da casca do caule é indicado no tratamento de gripes e bronquites.

\section{Boraginaceae}

Cordia leucocephala Moric. [moleque-duro, negroduro]

O decocto das folhas é usado contra indigestões e o das flores contra reumatismos e artrites. As partes aéreas, como "lambedor" (xarope), são empregadas como tônico geral.

Cordia multispicata Cham. [maria-preta]

O decocto das folhas e partes aéreas, tomado três vezes ao dia, é utilizado contra reumatismos e artrites.

\section{Bromeliaceae}

Bromelia laciniosa Mart. ex Schult.f. [macambira]

O decocto das raízes é empregado no tratamento de hepatites. As folhas, em forma de pó, e adicionadas aos alimentos são usadas como tônico e fonte de proteínas.
Tillandsia recurvata (L.) L. [barba-de-velho]

O infuso ou decocto da planta inteira é indicado contra reumatismos, úlceras e hemorróidas.

Tillandsia streptocarpa (L.) Baker [gravatá-do-ar]

Usa-se o decocto da planta inteira como purgativo, laxativo e emético.

\section{Burseraceae}

Commiphora leptophloeos (Mart.) J.B.Gillett [imburana, umburana]

O decocto ou xarope (lambedor) da casca do caule é empregado no tratamento de gripes, tosses e bronquites.

\section{Cactaceae}

Cereus jamacaru DC. [mandacaru]

Usa-se o infuso das raízes contra doenças respiratórias e renais, principalmente como diurético. A polpa do caule, misturada ao açúcar, é usada no tratamento de úlceras estomáquicas.

Melocactus zehntneri (Britton \& Rose) Luetzelb. [coroa-de-frade]

A polpa do caule, misturada ao açúcar ou mel, é indicada no tratamento de bronquites, tosses e debilidade física.

\section{Capparaceae}

Capparis jacobinae Moric ex Eichler [icó]

Emprega-se o decocto da casca do caule contra tosses e coqueluches. O decocto das raízes é usado contra a falta de menstruação.

\section{Celastraceae}

Maytenus rigida Mart. [bom-nome]

O decocto, infuso ou macerado da casca do caule é indicado contra as inflamações e infecções renais e ovarianas. O decocto da casca do caule também é empregado externamente no tratamento de úlceras externas.

\section{Chrysobalanaceae}

Licania rigida Benth. [oiticica]

Emprega-se o decocto ou macerado das folhas no tratamento do diabetes.

\section{Combretaceae}

Combretum leprosum Mart. [mofumbo]

As folhas e cascas do caule, em decocto ou 
xarope, são usadas como expectorante, contra tosses e coqueluches.

\section{Convolvulaceae}

Ipomoea asarifolia (Desr.) Roem. \& Schult. [salsa, salsa-do-rio]

O decocto das folhas e partes aéreas, em banhos e lavagens, é usado externamente, contra dermatites, escabioses, sífilis e úlceras externas.

Operculina hamiltonii (G. Don) D.F. Austin [batatade-purga]

Usa-se o tubérculo, preparado como xarope, contra bronquites e tosses. O decocto ou infuso, após as refeições, é empregado como laxativo, purgativo e carminativo. O macerado do tubérculo é usado como antiinflamatório e também contra as dores reumáticas.

Operculina macrocarpa (L.) Urb. [batata-de-purga]

O tubérculo é usado com as mesmas indicações e modo de usar de O. macrocarpa.

\section{Cucurbitaceae}

Luffa operculata (L.) Cogn. [cabacinha]

Usa-se o decocto de uma pequena porção do fruto contra amenorréias e no tratamento das sinusites.

\section{Euphorbiaceae}

Cnidoscolus infestus Pax. \& K. Hoffm. [urtiga-deboi, urtiga-branca]

As raízes, em decocto, são empregadas como antiinflamatório do trato urinário e ovariano.

\section{Cnidoscolus quercifolius Pohl [favela]}

Usa-se o decocto ou macerado da casca do caule contra inflamações dos ovários e próstatas.

Jatropha molissima (Pohl) Baill. [pinhão-bravo]

O látex ingerido “in natura” é empregado para tratar picadas de cobra. O óleo das sementes é usado como vermífugo veterinário.

Jatropha ribifolia (Pohl) Baill. [pinhão-de-purga]

O látex é empregado como sucedâneo e com mesmas indicações da espécie anterior.

Manihot glaziovii Müll. Arg. [maniçoba]

As folhas, aquecidas e aplicadas diretamente sobre a parte afetada, são usadas contra reumatismos.

\section{Fabaceae-Caesalpinioideae}

Bauhinia cheilantha (Bong.) Steud. [mororó, patade-vaca]

O decocto ou macerado da casca do caule é empregado como tônico, depurativo e no tratamento do diabetes.

Caesalpinia pyramidalis Tul. [catingueira]

O macerado do caule, misturado em vinho ou cachaça, é indicado como afrodisíaco. Preparado como decocto, é usado contra disenterias, diarréias e como estomáquico. O xarope é empregado como expectorante e indicado contra bronquites e tosses.

Senna martiana (Benth.) H. Irwin \& Barneby [sena, sene]

O infuso das folhas, tomado antes de dormir, é indicado como laxativo.

Senna spectabilis var. excelsa (Schrad.) H.S. Irwin \& Barneby [canafístula]

O decocto da casca do caule ou raiz é empregado contra gripes e tosses. As folhas em infusão ou decocção são indicadas como laxativo, purgativo e contra amenorréias. As sementes torradas e moídas, preparadas em infuso como café, são indicadas contra anemias.

\section{Fabaceae-Faboideae}

Amburana cearensis (Allemão) A.C. Sm. [cumaru, amburana-de-cheiro]

Um decocto ou xarope da casca do caule é utilizado no tratamento de gripes, tosses e bronquites. Uma maceração da casca do caule, no vinho ou cachaça, é indicada como tônica, no tratamento da anorexia. A casca seca, triturada em forma de pó, é aplicada contra úlceras externas. Em maceração dos frutos n’água é usada no tratamento de infecções urinárias. As sementes e as cascas torradas, posteriormente trituradas, reduzidas a pó, são usadas como "rapé" e empregadas no tratamento de sinusites.

Dioclea grandiflora Mart. ex Benth. [mucunã]

O decocto ou macerado das raízes é indicado no tratamento de inflamações prostáticas.

Erythrina velutina Willd. [mulungú]

O decocto ou infuso da casca do caule é indicado no tratamento das insônias, tosses e como vermífugo. Os frutos secos, triturados e adicionados ao tabaco, são usados como cigarros contra dor-de-dente. 
Zornia brasiliensis Vogel [urinária, urinana]

Um macerado da planta inteira é empregado como diurético e contra doenças venéreas.

\section{Fabaceae-Mimosoideae}

Anadenanthera colubrina var. cebil (Griseb.) Altschul [angico]

O decocto ou xarope da casca do caule é empregado no tratamento de tosses, coqueluches e bronquites. Uma maceração dos n’água, são indicados contra insônias e utilizados como sedativos.

Enterolobium contortisiliquum (Vell.) Morong [timbaúba, tambor, orelha-de-negro]

O decocto da casca do caule é usado como antiinflamatório. Os frutos são usados como "shampoo" contra escabioses.

Mimosa ophthalmocentra Mart. ex Benth. [jurema]

Usa-se o decocto ou xarope da casca do caule contra bronquites e tosses.

Mimosa tenuiflora (Willd.) Poir [jurema]

O mesmo uso e indicações que a espécie anterior.

\section{Lamiaceae}

Hypenia salzmannii (Benth.) Harley [canela-deurubu]

O decocto, infuso ou xarope das folhas é empregado no tratamento de gripes, tosses e doenças respiratórias.

Hyptis suaveolens (L.) Poit. [alfazema-de-caboclo, alfavaca-brava]

$\mathrm{O}$ infuso ou decocto das folhas e flores é indicado contra dismenorréias, doenças respiratórias, febres e indigestões. As flores secas são usadas como cigarros e empregadas contra dores de dentes e cefaléias. As sementes, colocadas diretamente nos olhos, são usadas para retirar impurezas.

Leucas martinicensis (Jacq.) R. Br. [cordão-de-frade, cordão-de-são-francisco]

O decocto das partes aéreas é usado contra inflamações e problemas renais. O cataplasma ou decocto das folhas é usado externamente em massagens contra as dores reumáticas.

\section{Loasaceae}

Aosa rupestris (Gardner)Weigend [urtiga-branca]

O decocto ou o macerado das raízes, em vinho ou cachaça, é empregado contra inflamações uterinas e prostáticas.

\section{Loganiaceae}

Spigelia anthelmia L. [arapabaca, lombrigueira]

Usa-se o infuso ou decocto da planta inteira como vermífugo.

\section{Malvaceae}

Ceiba glaziovii (Kuntze) K. Schum. [barriguda]

O decocto das folhas é usado, externamente, contra reumatismos e edemas.

\section{Melochia tomentosa L. [malva-roxa]}

O decocto ou xarope das folhas é usado contra tosses e bronquites.

\section{Sida galheirensis Ulbr. [malva-branca]}

Usam-se as partes aéreas, em decocto ou xarope, contra tosses e coqueluches.

Sidastrum micranthum (A. St.-Hil.) Fryxell [guaxima, malva-preta]

O infuso das folhas é usado contra bronquites, tosses e asmas. As folhas são usadas em cataplasmas, com manteiga quente ou óleo de oliva, como emoliente.

\section{Passifloraceae}

Passiflora foetida L. [camapu, maracujá-de-estalo]

O decocto ou xarope da planta inteira é usado contra blenorréias. As folhas são empregadas contra hemorróidas.

\section{Papaveraceae}

Argemone mexicana L. [cardo-santo]

As sementes, em infuso ou decocto, são indicadas como purgativas, laxativas e digestivas. O látex, usado diretamente nos olhos, é indicado contra conjuntivites.

\section{Rhamnaceae}

Ziziphus cotinifolia Reiss. [juazeiro]

A casca do caule, reduzida a pó, é usada como dentifrício nas escovações diárias. Em raspas, são usadas contra caspas e seborréias, substituindo o shampoo. Em xaropes ou "garrafadas" é usada no tratamento das tosses.

Ziziphus joazeiro Mart. [juazeiro]

Possui as mesmas indicações e usos que a espécie anterior. 


\section{Rubiaceae}

Guettarda angelica Mart. ex Müll. Arg. [angélicabrava]

Usa-se o infuso ou decocto das raízes contra cólicas menstruais, constipações e febres.

Tocoyena formosa (Cham. \& Schltdl.) K. Shum. [jenipapim, jenipapo-bravo]

As folhas quentes são usadas em cataplasmas, com óleo de oliva ou manteiga, contra as dores reumáticas.

\section{Sapindaceae}

Cardiospermum halicacabum L. [para-tudo]

A planta inteira, em infuso ou decocto, é indicada contra problemas hepáticos e reumatismos, como tônica, diurética e emenagoga.

\section{Sapotaceae}

Sideroxylon obtusifolium (Roem. \& Schult.) T.D. Penn. subsp. obtusifolium [quixaba, rompe-gibão]

Emprega-se a casca do caule, em decocto ou macerado, no tratamento das inflamações ovárianas e do diabetes.

\section{Selaginellaceae}

Selaginella convoluta (Arnott) Spreng [mão-fechada, jericó]

Usa-se o decocto ou macerado da planta inteira como afrodisíaco, diurético e contra amenorréias.

\section{Solanaceae}

Nicandra physalodes (L.) Gaertn. [quintilho]

Emprega-se o decocto da planta inteira como calmante.

Nicotiana glauca Graham [fumo-bravo, charuto-do-rei]

As folhas secas, usadas como cigarros, são indicadas no tratamento de asmas e enxaquecas.

Physalis angulata L. [camapu, bate-testa]

Oinfusodasfolhaséindicadocomoantiinflamatório da bexiga e dos rins, e como sedativo. A planta inteira, em decocto, é empregada externamente contra dermatites e otites.

Solanum agrarium Sendtn. [gogóia]

Usa-se o decocto das raízes como abortivo e contra inflamações prostáticas.

Solanum rhytidoandrum Sendtn. [jurubeba-branca]

Emprega-se o decocto das raízes contra doenças do fígado.
Solanum paniculatum L. [jurubeba, jurubeba-roxa]

As raízes, em decocto, são indicadas como diuréticas e contra as doenças do fígado. O suco do fruto fresco é usado como tônico contra anemias e tuberculoses.

\section{Turneraceae}

Turnera chamaedrifolia Cambess. [chanana]

O decocto das raízes éindicado contra amenorréias e dismenorréias. A planta inteira, como xarope, é usada como expectorante, contra bronquites e tosses.

\section{Verbenaceae}

Lippia microphylla Cham. [alecrim-pimenta]

O decocto ou xarope das folhas é usado como anti-séptico das vias nasais e contra doenças respiratórias.

\section{Violaceae}

Hybanthus calceolaria (L.) Schulze-Menz [ipecacoanha, papaconha]

O decocto ou xarope das raízes é indicado contra a ausência de menstruação. Reduzidas a pó, misturadas com amido e preparadas como pílulas, são indicadas no tratamento de diarréias e amebíases.

\section{CONCLUSÕES}

Os dados obtidos das 70 espécies, usadas na medicina popular do Cariri Paraibano, indicam que cerca de treze espécies são as mais conhecidas e encontradas em todos os raizeiros e vendedores de plantas medicinais nos mercados populares visitados. A maioria das plantas comercializadas e empregadas com fins medicinais, no dia a dia da população local, é constituída de espécies nativas. Os raizeiros e vendedores possuem considerável conhecimento das plantas medicinais e seus usos, para uma ampla variedade de doenças, pela experiência adquirida de gerações anteriores.

AGRADECIMENTOS: Ao Conselho Nacional de Pesquisa e Desenvolvimento $(\mathrm{CNPq})$ pelo apoio financeiro e bolsas concedidas; ao Dr. José Maria Barbosa-Filho e Dra. Maria Regina Barbosa, curadora do Herbário JPB, pelos apoios pessoais e institucionais; ao Dr. Jnanabrata Bhattacharyya pela revisão do abstract; à Dulce Gonçalves de Oliveira pelo apoio técnico. 


\section{REFERÊNCIAS}

AGRA, M.F. 1996. Plantas da medicina popular dos Cariris Velhos, Paraíba, Brasil. A União, João Pessoa. 125p.

AGRA, M.F.; ROCHA, E.A.; FORMIGA, S.C. \& LOCATELLI, E. 1994. Plantas medicinais dos Cariris Velhos, Paraíba, parte I: subclasse Asteridae. Revista Brasileira de Farmácia, 75(3): 61-64.

AGRA, M.F.; LOCATELLI, E.; ROCHA, E.A.; BARACHO, G.S. \& FORMIGA, S.C. 1996. Plantas medicinais dos Cariris Velhos, Paraíba, Brasil, parte II: subclasses Magnoliidae, Caryophyliidae, Dilleniidae e Rosidae. Revista Brasileira de Farmácia, 77(3): 97-102.

AGRA, M.F.; BARACHO, G.S.; NURIT, K.; BASÍLIO, I.J.L.D. \& COELHO, V.P.M. 2007. Medicinal and poisonous diversity of the flora of "Cariri Paraibano", Brazil. Journal of Ethnopharmacology, 111(2): 383-395.

ANDRADE-LIMA, D. 1981. The caatinga dominium. Revista Brasileira de Botânica, 4(2): 149-153.

APG II. 2003. An update of the Angiosperm Phylogeny Group Classification for the orders and families of flowering plants: APG II. Botanical Journal of the Linnean Society, 141: 399436.

BARACHO, G.S. \& AGRA, M.F. 1995. Etnomedicina da família Malvaceae nos Cariris Velhos, Paraíba, Brasil. Revista Brasileira de Farmácia, 76(2): 48-52.

BASÍLIO, I.J.L.D.; SILVA, K.N.; BARACHO, G.S. \& AGRA, M.F. 2003. Caracterização morfo-anatômica de Spigelia anthelmia L. (Loganiaceae), espécie da medicina popular na Paraíba, Brasil. Revista Nordestina de Biologia, 17(1/2): 1122.

BRUMMITT, R.K. \& POWELL, C.E. 1992. Authors of plant names. Royal Botanic Gardens, Kew, Great Britain. 732p.

CARVALHO, F.A.F. \& CARVALHO, M.G.F. 1985. Vegetação. Pp 34-43. In: C.A.G. Egler, E.C.P. Dália \& N. Gedanken, (orgs.), Atlas geográfico do Estado da Paraíba. Grafset, João Pessoa. 99p.

COELHO, V.P.M.; AGRA, M. F. \& BARBOSA, M. R. V. 2006. Estudo farmacobotânico das folhas de Tocoyena formosa (Cham. \& Schltdl.) K. Schum. (Rubiaceae). Revista Brasileira de Farmacognosia, 16: 170-177.

LEAL, C.K.A. \& AGRA, M.F. 2005. Estudo farmacobotânico comparativo das folhas de Jatropha molissima (Pohl) Baill. e Jatropha ribifolia (Pohl) Baill. (Euphorbiaceae). Acta Farmaceutica Bonaerense, 24(1): 5-13.

SILVA, K.N. \& AGRA, M.F. 2002. Etnomedicina e farmacobotânica das Passifloraceae da caatinga paraibana, Brasil (Passifloraceae). Pp 33-45. In: M.F. Vanderlei, (org.),
Iniciados 8. EDUFPB, Universidade Federal da Paraíba, João Pessoa. 295p.

SILVA, K.N.; AGRA, M.F. \& BARACHO, G.S. 2002. Estudo Etnomedicinal e Farmacobotânico Comparativo entre Passiflora foetida L. e Passiflora cincinnata Mast. (Passifloraceae). Revista Brasileira de Farmácia, 83(1/4): 51-55.

SILVA, S.A.S.; RODRIGUES, M.S.L.; AGRA, M.F., DACUNHA, E.V.L.; BARBOSA-FILHO, J.M. \& SILVA, M.S. 2004. Flavonoids from Cordia globosa. Biochemical Systematics and Ecology, 32(3): 359-361.

Submetido em 23/07/2007

Aceito em 05/10/2007 